\title{
Analysis on the Propagation of the Fiber-0ptic Signals in the Perturbed Nonlinear Schrödinger Equation
}

\author{
Qianqian Xing, Jiuli Yin*, Lixin Tian \\ Nonlinear Scientific Research Center, Faculty of Science, Jiangsu University, Zhenjiang, China \\ Email: ${ }^{*}$ yil@ujs.edu.cn
}

Received 27 August 2014; revised 7 October 2014; accepted 10 November 2014

Copyright (C) 2014 by authors and OALib.

This work is licensed under the Creative Commons Attribution International License (CC BY).

http://creativecommons.org/licenses/by/4.0/

(c) (i) Open Access

\begin{abstract}
Chaos appears in the whole process of fiber-optic signal propagation with one external perturbation due to the absence of damping. Via adding a proper controller, chaos cannot be suppressed when the controller's strength is weak. With the increase of the controller strength, the fiber-optic signal can stay in a stable state. However, unstable phenomenon occurs in the propagation of the fiber-optic signal when the strength exceeds a certain degree. Moreover, we discuss the parameters' sensitivity to be controlled. Numerical results show that vibration, oscillation and escape can occur during the transmission of optic signals with different parametric regions.
\end{abstract}

Keywords

Fiber-Optic Signal, Nonlinear Schrödinger Equation, Vibration, Oscillation, Escape

Subject Areas: Applied Physics, Partial Differential Equation

\section{Introduction}

The nonlinear Schrödinger equation

$$
i u_{t}+u_{x x}+a u|u|^{2}=0
$$

is widely used in many areas of physics, such as the evolution of nearly monochromatic, high intensity laser beam propagation and one-dimensional waves in deep wave. It also describes the evolution of the slowly varying envelope of an optical plus [1]-[3]. The NLS equation plays an important role in understanding optic fibers which is of importance to the fiber-based telecommunications [4]. This paper is devoted to the application of

*Corresponding author.

How to cite this paper: Xing, Q.Q. Yin, J.L. and Tian, L.X. (2014) Analysis on the Propagation of the Fiber-Optic Signals in the Perturbed Nonlinear Schrödinger Equation. Open Access Library Journal, 1: e721. http://dx.doi.org/10.4236/oalib.1100721 
system of Equation (1.1) in optic fiber-based telecommunications, where $x$ represents the non-dimensional distance along the fiber-optic, $t$ represents time in a dimensionless form, and $a$ is a real valued constant. The dependent variable function $u(x, t)$ is a complex valued function that represents the wave profile. The instabilities and chaos of Equation (1.1) have been studied in [8] [9].

The NLS equation is completely integrable. Many studies have been devoted to the system of Equation (1.1) because its characteristics might be changed under small perturbation [5]-[7] [10]. In fact, the transmission of fiber-optic signal will be disturbed inevitably and the signal propagation cannot exist in pure environment; it is always influenced by external environmental perturbations. In some certain conditions, the process of fiber-optic signal transmission can be depicted as the following equation,

$$
i u_{t}+u_{x x}+a u|u|^{2}=d \cos (\omega x) \exp (i c t),
$$

where $d$ and $w$ are the amplitude and frequency of a certain perturbation respectively; $c$ is the coefficient of linear term.

We focus on the following two points: The first problem is how to design a controller to suppress chaos in system of Equation (1.2). We can observe that there is no damping in the system of Equation (1.2). Once perturbed with external forcing, chaos may be occurred in system of Equation (1.2). Therefore, we will select a controller which has the same function with the damping.

The second interesting problem is to analyze the parameter regions for optical fiber signals stable propagation of the controlled system. That is, we will discuss the parameters' sensitivity to be controlled. For example, parameters of system of Equation (1.1) are associated with the properties of optic-fibers media, so we can obtain the preferable media by studying the region of parameter to reduce the influence of perturbation in the process of optic fiber signal propagation.

In this paper, the system of Equation (1.2) is investigated in detail. We study the dynamical behaviors by the fourth-order Runge-Kutte method. Maximum Lyapunov exponents and bifurcation diagrams are used to show the behaviors of the system of Equation (1.2) in some certain parametric region. The organization of the paper is as follows. In Section 2, we study the chaotic behavior of system of Equation (1.2) and the chaos can be controlled by finding an appropriate controller. In Section 3, we study the chaos control in controlled system by Melnikov method [11]-[15]. In Section 4, the bifurcation diagrams and the maximum Lyapunov exponents are given to support the theoretical analysis. Last section is the conclusion.

\section{Analysis of Perturbed System}

Supposing $u=\varphi(x) \mathrm{e}^{i c t}$ and substituting $u=\varphi(x) \mathrm{e}^{\text {ict }}$ into Equation (1.2), one can get an elimination of the time dependence as follows:

$$
\phi^{\prime \prime}+a \phi^{3}+c \phi=d \cos (\omega x),
$$

where $d, w$ denote the amplitude and the frequency of the parametric perturbation, respectively. Here $d$ and $w(\geq 0)$ are real parameters.

We make the transformation $\phi \rightarrow x_{1}, \phi^{\prime} \rightarrow x_{2}$, then Equation (2.1) can be transformed into first-order nonautonomous equations

$$
\left\{\begin{array}{l}
x_{1}^{\prime}=x_{2}, \\
x_{2}^{\prime}=-a x_{1}^{3}+c x_{1}+d \cos (\omega x) .
\end{array}\right.
$$

Next we will consider the chaotic behavior of system of Equation (2.2). The study is carried out by taking $\alpha=1, \quad c=1, \omega=0.05$ and setting $d$ as the variable with the initial condition [1.0,0.0].

According to the bifurcation diagram and the maximum Lyapunov exponents (see Figure 1). We can observe that the value of Lyapunov exponents is positive, so the system easily converts to chaos even if there is a small perturbation. Because the system in the chaotic state is very sensitive to its initial condition and chaos often causes irregular behavior, chaos is undesirable. So an appropriate controller is needed to satisfy the practical applications of fiber-optic communication.

It is not difficult to find that system of Equation (2.2) is similar to the duffing system, except the damping term is absent in Equation (2.2). Therefore, we will select a controller that have the same function with the damping. 


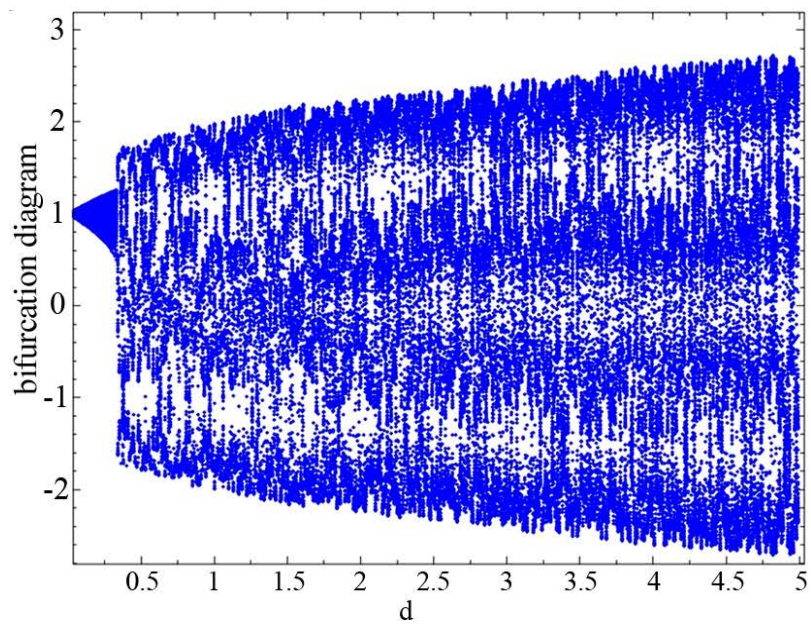

(a)

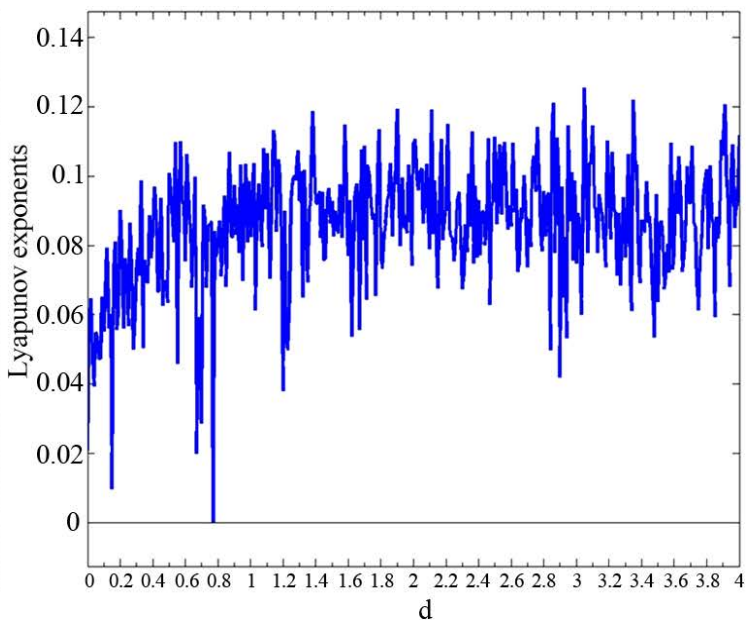

(b)

Figure 1. (a) Bifurcation diagram of $x \quad(d \in[0,5])$ and (b) the maximum Lyapunov exponents corresponding to (a).

We modify the perturbed system of Equation (2.2) to a controlled system as follows

$$
i u_{t}+u_{x x}+a u|u|^{2}=d \cos (\omega x) \exp (i c t)+\varepsilon\left(k_{1} u+k_{2} u_{x}\right),
$$

where $k_{1}, k_{2}$ and $\varepsilon$ are constants. Here $\varepsilon$ denotes the controller's strength and the controller $k_{1} u+k_{2} u_{x}$ has the function of damping.

Suppose $u=\varphi(x) \mathrm{e}^{\text {ict }}$ and substitute it into (2.3), then Equation (2.3) takes the form as follows:

$$
\phi^{\prime \prime}+a \phi^{3}+c \phi=d \cos (\omega x)+\varepsilon\left(k_{1} \phi+k_{2} \phi^{\prime}\right) .
$$

So (2.4) can be written as the following form

$$
\left\{\begin{array}{l}
x_{1}^{\prime}=x_{2} \\
x_{2}^{\prime}=-\alpha x_{1}^{3}+c x_{1}+d \cos (\omega x)+\varepsilon\left(k_{1} x_{1}+k_{2} x_{2}\right) .
\end{array}\right.
$$

Now we will study the controlled system of Equation (2.5) with $\alpha=1, c=1, d=2, \omega=0.05, k_{1}=0.1$ and $k_{2}=-2$. According to the bifurcation diagram and the maximum Lyapunov exponents in Figure 2, we can obtain that chaos occur within $\varepsilon \in(0,0.04)$ for the controller being too weak to inhibit the chaos. The perturbed system of Equation (2.4) can be suppressed to the stable station with the larger $\varepsilon$. Moreover we cannot ignore the phenomenon that some limit cycles are symmetric about origin when $\varepsilon>11.7$, while it is not symmetric when $\varepsilon>11.7$. It is easy to find that the signal cannot propagate normally and might leak from the media, which is called escape.

Remark 1. According to above analysis, the increase of the controller's coefficient $\varepsilon$ makes the system stable, but the signal escape when $\varepsilon$ cross a certain value.

\section{Melnikov Analysis for the Perturbed System}

In this section, the chaotic thresholds of Equation (2.5) can be characterized by Melnikov theory [14] [15]. The complex dynamics of stable oscillators can be easily depicted. Moreover, the necessary conditions for the occurrence of homoclinic bifurcation can be found.

When $\varepsilon=0$, Equation (2.5) is an unperturbed system, that is,

$$
\left\{\begin{array}{l}
x_{1}^{\prime}=x_{2}, \\
x_{2}^{\prime}=-\alpha x_{1}^{3}+c x_{1} .
\end{array}\right.
$$

Obviously, the unperturbed equation has the following three equilibrium points $O_{1}(0,0), C_{1}\left(-\sqrt{\frac{c}{a}}, 0\right)$ and 


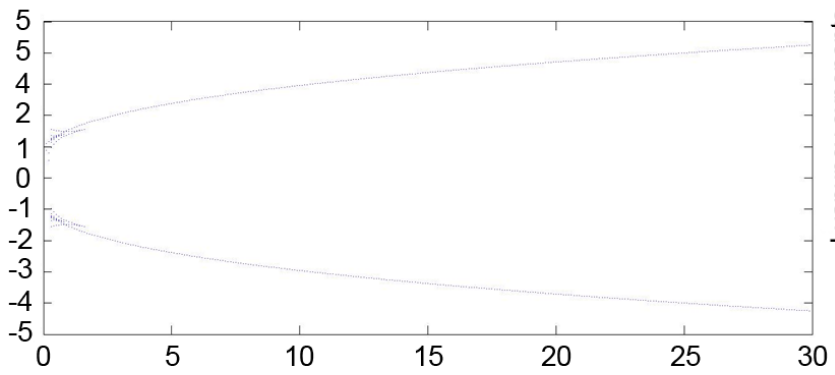

(a)

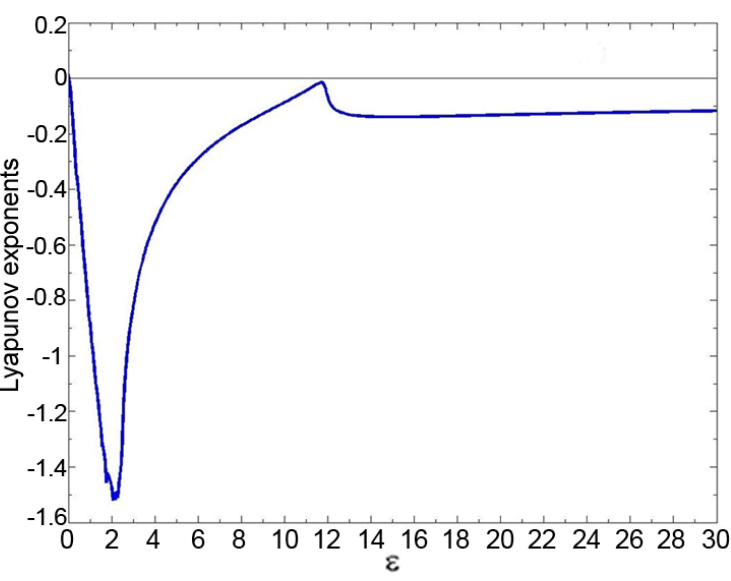

(b)

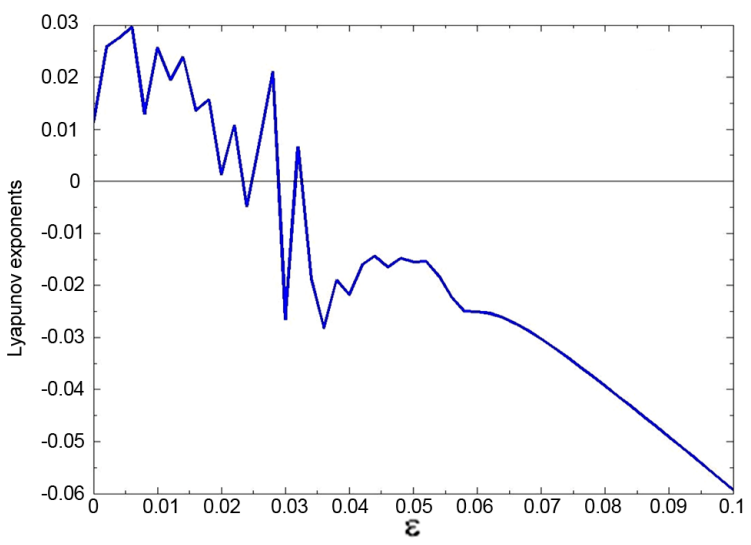

(c)

Figure 2. Bifurcation diagram and Lyapunov exponents. (a) Bifurcation diagram of $x \quad(\varepsilon \in[0,30])$; (b) The maximum Lyapunov exponents corresponding to (a); (c) Enlarge view of (b).

$C_{2}\left(\sqrt{\frac{c}{a}}, 0\right)$ on the $x_{1}$-axis.

The system of the unperturbed Equation (2.5) corresponds to a planar Hamiltonian system with a potential function

$$
V\left(x_{1}\right)=-\frac{c}{2} x_{1}^{2}+\frac{a}{4} x_{1}^{4} .
$$

And the associated Hamiltonian function is

$$
H\left(x_{1}, x_{2}\right)=-\frac{c}{2} x_{1}^{2}+\frac{a}{4} x_{1}^{4}+\frac{x_{2}^{2}}{2}=h,
$$

where $h$ is a constant. As we know, for a fixed $h$, the Hamiltonian function (3.2) determines a set of invariant curves of Equation (3.1). With $h$ varying, the function (3.2) determines different families of orbits of Equation (3.1) which corresponds to different dynamical behaviors. Via the determinant of the Jacobian matrix, the stability of the equilibrium points can be obtained, which the potential function and the phase portrait are given in Figure 3(a) and Figure 3(b) with $a=1, c=4$.

The unperturbed system of Equation (3.1) has close orbits. However, the close orbits break with adding the perturbation. Because the nonlinear dispersive system of Equation (3.1) is very sensitive to its initial values and chaos often occurs and causes irregular behaviors. When adding the external periodic perturbation, the closed homoclinic orbits break, and may transverse homoclinic orbits. 


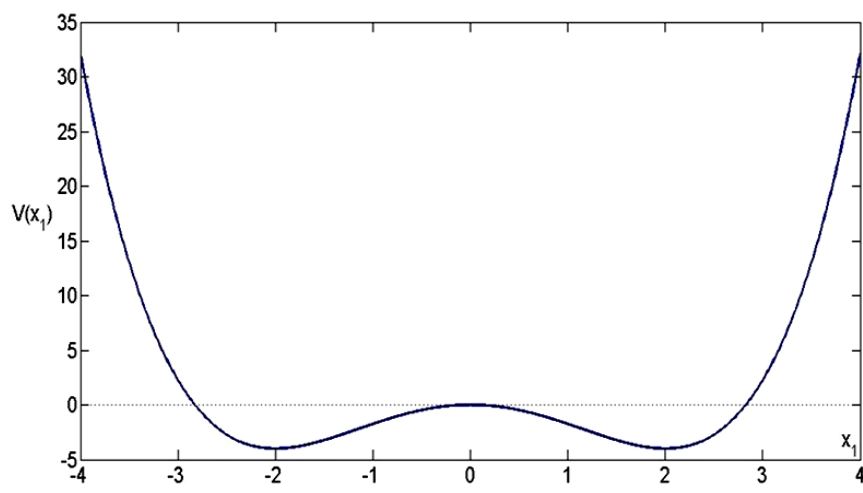

(a)

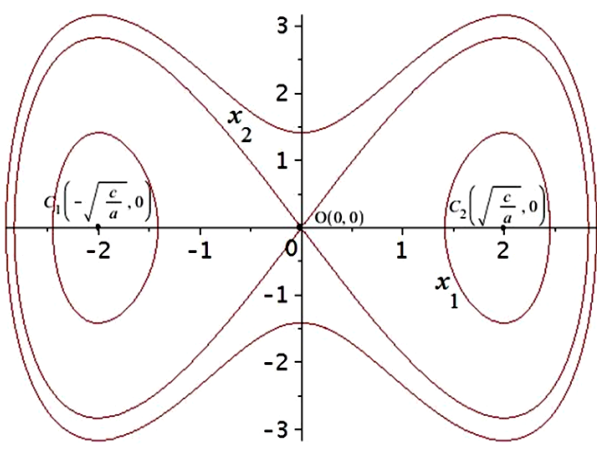

(b)

Figure 3. (a) The potential function and (b) phase portrait of system of Equation (3.1) with $a=1, \quad c=4$.

We suppose that the unperturbed homoclinic orbits are written as $\left(x_{0}, y_{0}\right)=\left(x_{1}^{ \pm}(\xi), x_{2}^{ \pm}(\xi)\right)$, and the Melnikov function can be given by

$$
M\left(\xi_{0}\right)=\int_{-\infty}^{\infty} x_{2}(\xi)\left[r \cos w\left(\xi+\xi_{0}\right)\right] \mathrm{d} \xi=-r \sin w \xi_{0} A,
$$

where $A=\int_{-\infty}^{\infty} x_{2}(\xi) \sin w \xi \mathrm{d} \xi, \quad \xi_{0}$ is the cross section time of the Poincaré map and $\xi_{0}$ can be interpreted with the initial time of the external perturbation. Because there exists a $\xi_{0}$ that $M\left(\xi_{0}\right)=0$. Thus, when Equation (3.1) subjected to the external forcing, chaos may occur.

We therefore apply the Melnikov method to system of Equation (2.2) for finding the criterion of the existence of homoclinic bifurcation and chaos. Compared with duffing system [12] [13], we found that the damping term is absent. To suppress chaos, we add the controller $\varepsilon\left(k_{1} x_{1}+k_{2} x_{2}\right)$ to the Equation (2.2).

For the controlled system of Equation (2.5), we note that $x_{2}(\xi)$ is a function of time from $-\infty$ to $+\infty$, then the Melnikov function can be given by:

$$
M\left(\xi_{0}\right)=\int_{-\infty}^{\infty} x_{2}(\xi)\left[-k x_{2}(\xi)+r \cos w\left(\xi+\xi_{0}\right)\right] \mathrm{d} \xi=-k B-r \sin w \xi_{0} A
$$

where $B=\int_{-\infty}^{\infty}\left[x_{2}(\xi)\right]^{2} \mathrm{~d} \xi, A=\int_{-\infty}^{\infty} x_{2}(\xi) \sin w \xi \mathrm{d} \xi$. Therefore, we discuss the chaotic threshold of the controlled system of Equation (2.5).

Thus, if

$$
k \leq k_{0}=\left|\frac{r A}{B}\right|
$$

the following theorem can be obtained.

The homoclinic bifurcation will occur at $k_{0}=R(r, w)$,

This implies that if $\varepsilon>0$ is sufficiently small, the transverse homoclinic orbits exist and system may be chaotic.

We use numerical simulations to support the theoretical results of the previous section and to seek the controllable area due to the complex expression of $k_{0}=R(r, w)$. There are several parameters, which play different and significant roles in disturbed and controlled system. Based on the above analysis, we can obtain chaotic threshold of the controlled system of Equation (2.5) shown in Figure 4. The numerical results show that the signal cannot propagate stably unless increasing the strength of controller. So the strength of controller should be selected the biggest value under the frequency. With the increase of $w$, the chaos can be well controlled.

\section{Parameters' Sensitivity to Be Controlled}

The controlled system has several parameters. Each of them plays different and virtual roles in the system. In this section, we will analyze the influence on optic-fiber signals propagation of controlled system of Equation (2.5) when the parameters of system of Equation (2.5) varying with the fixed controller. The second interesting problem is to analyze the parameter regions for optical fiber signals stable propagation of the controlled system. 


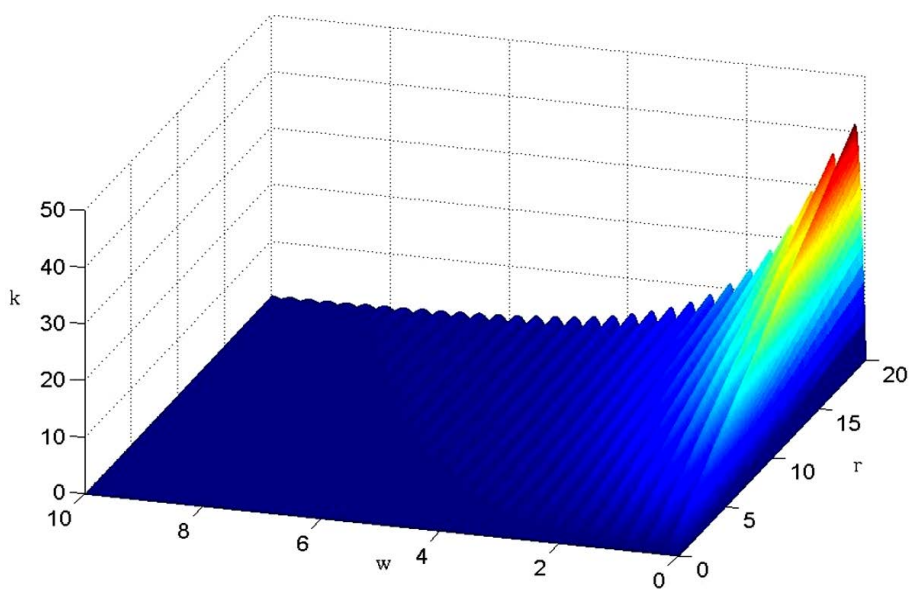

Figure 4. Chaotic threshold of the system of Equation (3.1) in $(r, w, k)$ plane with $a=1, c=1$.

The bifurcation parameters are considered in four cases: i) setting $\alpha=1, \varepsilon=2, c=1, k_{1}=0.1$ and $k_{2}=-2$, the parameter $\varepsilon d$ is varying in range $0 \leq \varepsilon d \leq 150$; ii) setting $\alpha=1, c=1, d=2, \varepsilon=2$, $k_{1}=0.1$ and $k_{2}=-2$; the parameter $w$ is varying in range $0 \leq w \leq 2.5$; iii) setting $\alpha=1, \omega=0.05$, $\varepsilon=2, d=2, k_{1}=0.1$ and $k_{2}=-2$, the parameter $c$ is varying in range $0 \leq c \leq 4$; iv) setting $c=1$, $\omega=0.05, \varepsilon=2, d=2, k_{1}=0.1$ and $k_{2}=-2$, the parameter $a$ is varying in range $0 \leq a \leq 4$.

Bifurcation diagrams and maximum Lyapunov exponents of system of Equation (2.5) are given in four cases (See Figures 5-7).

For case i), the bifurcation diagrams of system Equation (2.5) in $(d, x)$ plane and maximum Lyapunov exponents are given in Figure 5. We can find that chaos can be suppressed to the stable state comparing with Figure 1. Moreover, there are two other obvious features: 1 ) The cycle is not symmetric about origin with $d \in(0,0.58)$; 2) Oscillation appears when $d>45.2$, which is distinct with the increase of amplitude.

Remark 2. The system can be controlled well when the perturbation's amplitude is not too large. With the increase of amplitude, it is difficult to control the fiber signal and oscillation occurs.

For case ii), the bifurcation diagrams of system of Equation (2.5) in $(\omega, x)$ plane and maximum Lyapunov exponents are given in Figure 6. It is not difficult to find that chaos in system of Equation (2.5) is suppressed. Besides we can get other two results: 1$)$ the limit cycle is symmetric about origin within $w \in(0,0.56)$; 2) escape appears as $\omega>0.56$.

Remark 3. The system can be controlled when the frequency of perturbation is not too large. With the increase of frequency, it is difficult to control. The fiber signal might be leaked from the media and escape appears.

For case iii), after studying the bifurcation diagram and maximum Lyapunov exponents in Figure 7, it is not difficult to obtain the follow results: i) The behavior of the system is very well, the chaos are suppressed are symmetric about origin as $c<1.58$; ii) There are obvious oscillation with $c \in(1.58,2.68)$; iii) Escapes appear as $c>2.68$.

Remark 4. The system can be controlled very well with $c$ in a certain range. It is not easy to control the system when $c$ exceed the range. And escape and vibrate of signals also occur.

For case iv), we can obtain the following results after studying the bifurcation diagram and maximum Lyapunov exponents in Figure 8. i) The behavior of the system is well, chaos is inhibited; ii) The cycle is not symmetric about origin with $a \in(0,0.085)$; iii) The behavior of system is cycle as $a>0.085$.

Remark 5. The system is stable despite $a$ changes in a wide range. We can deduce that the influence of nonlinear term on system is weaker.

\section{Conclusion}

Inspired by the research of the duffing system [11]-[13], we notice that chaos may occur easily in this system due to the absence of damping in system of Equation (1.2). This phenomenon will cause the distortion in the 


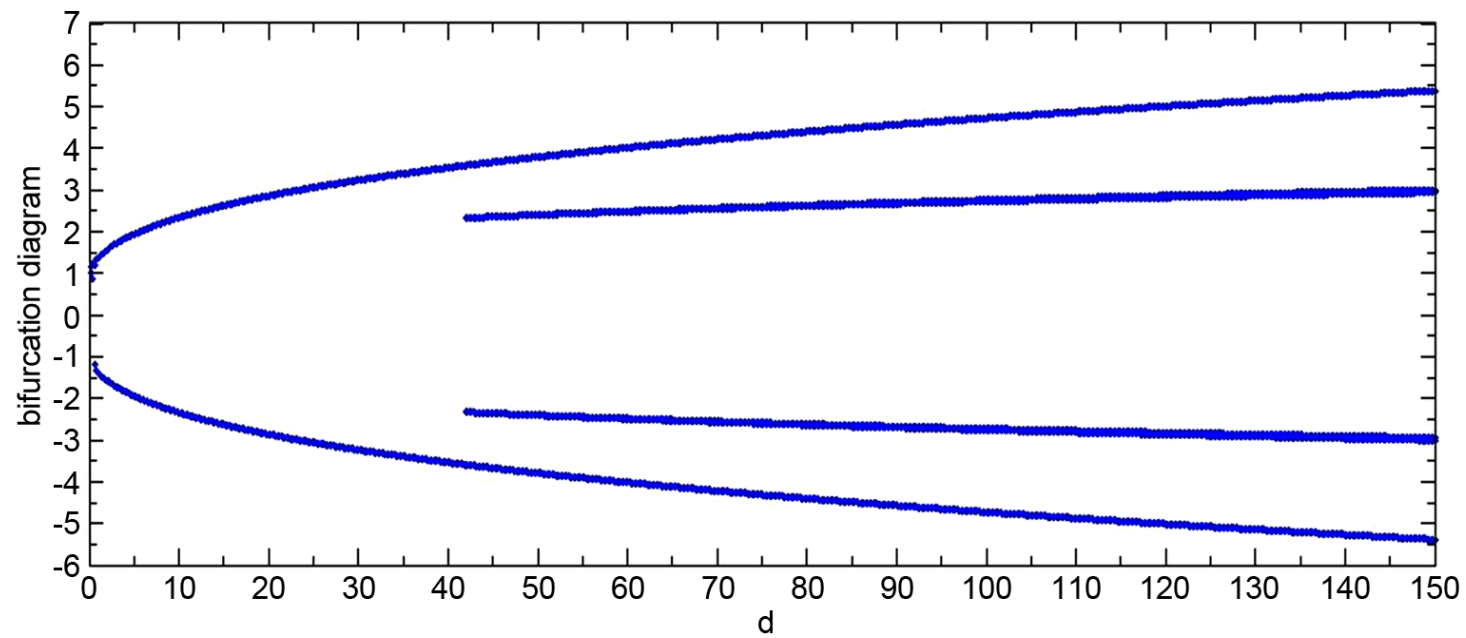

(a)

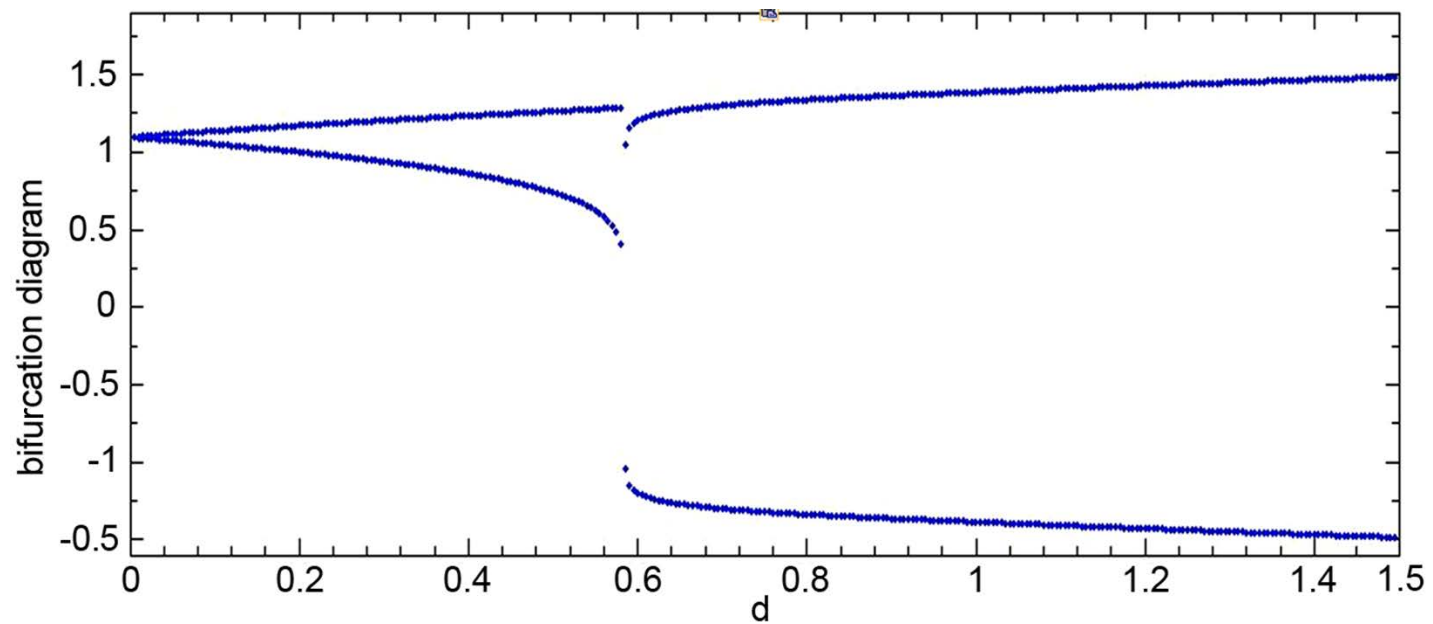

(b)

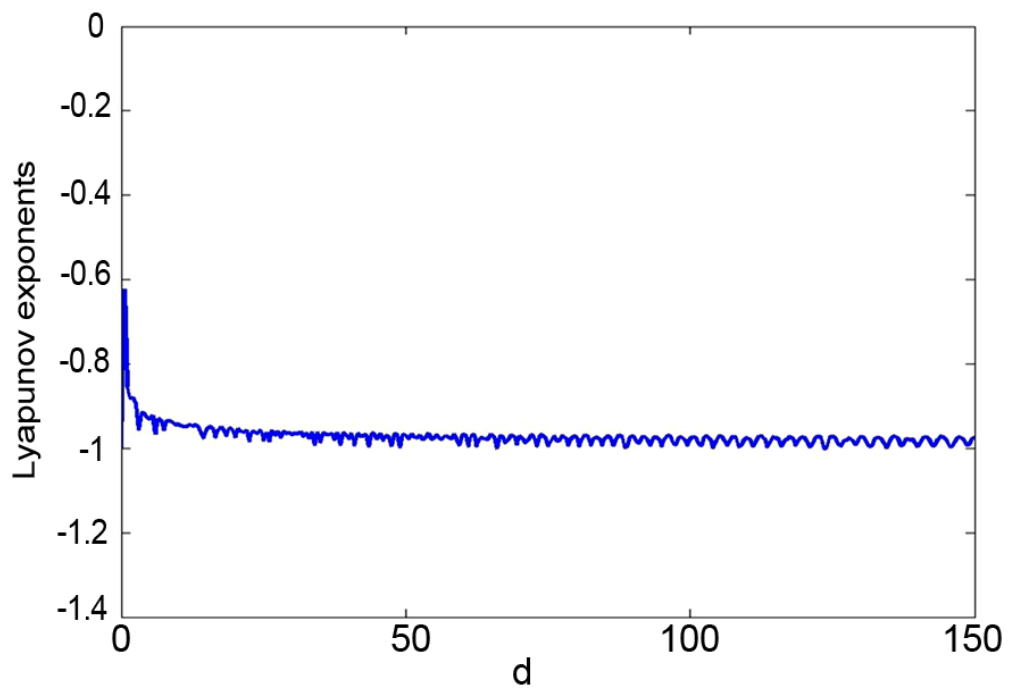

(c)

Figure 5. Bifurcation diagram and the maximum Lyapunov exponents spectrum. (a) Bifurcation diagram of $x$ $(d \in[0,150])$; (b) Enlarging view of (a); (c) Maximum Lyapunov exponents corresponding to (a). 


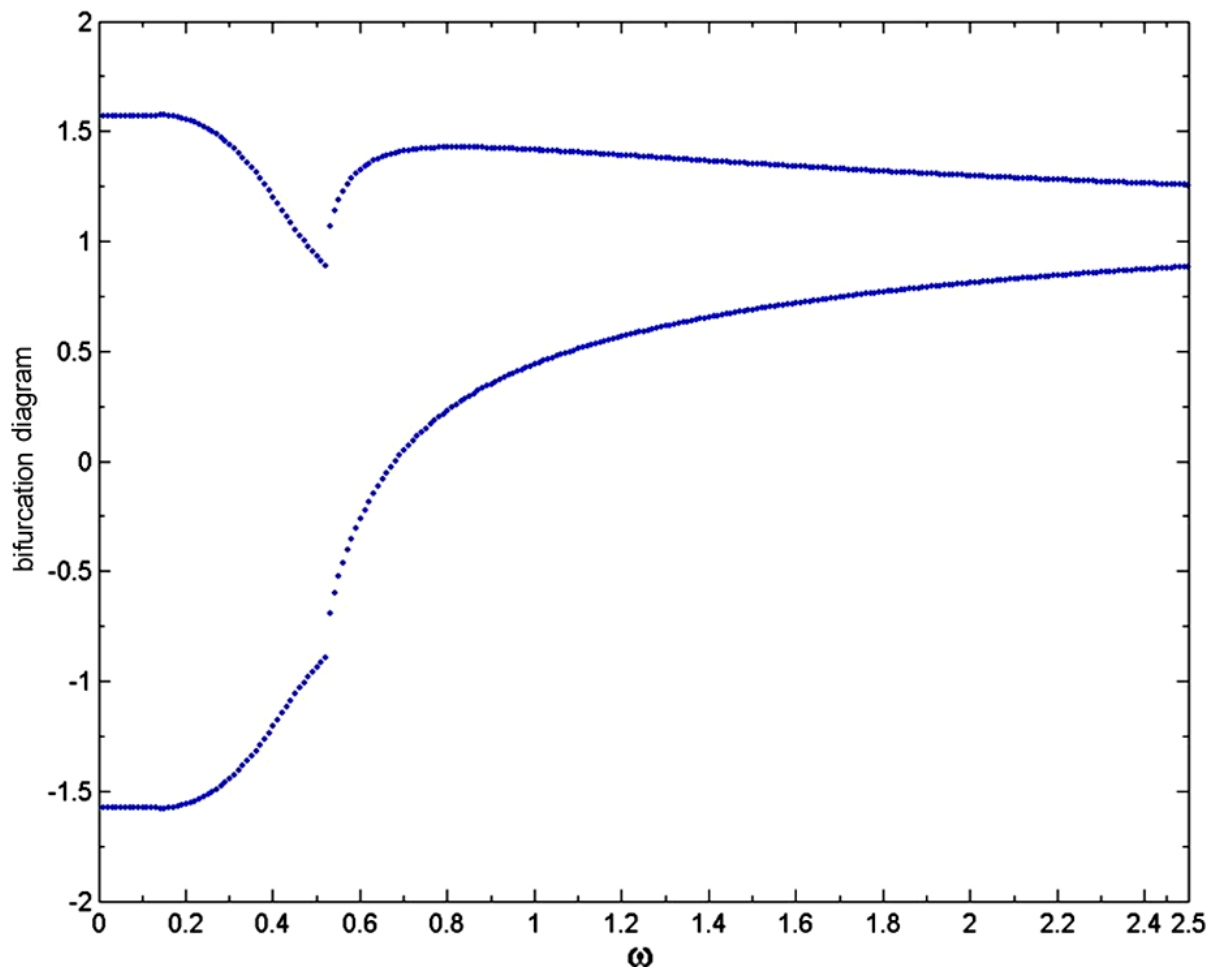

(a)

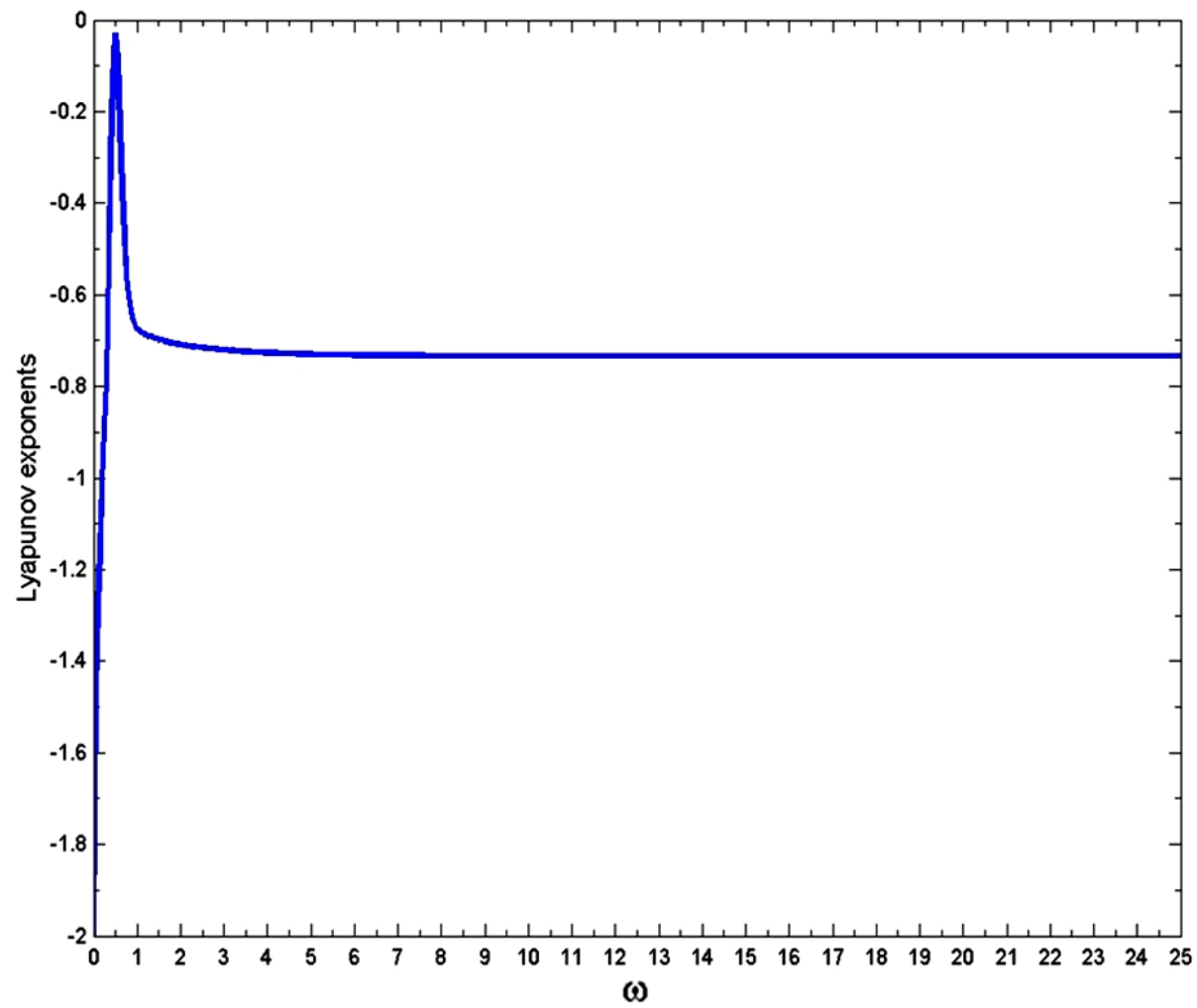

(b)

Figure 6. (a) Bifurcation diagram of $x \quad(\omega \in[0,2.5])$; (b) Maximum Lyapunov exponents corresponding to (a). 


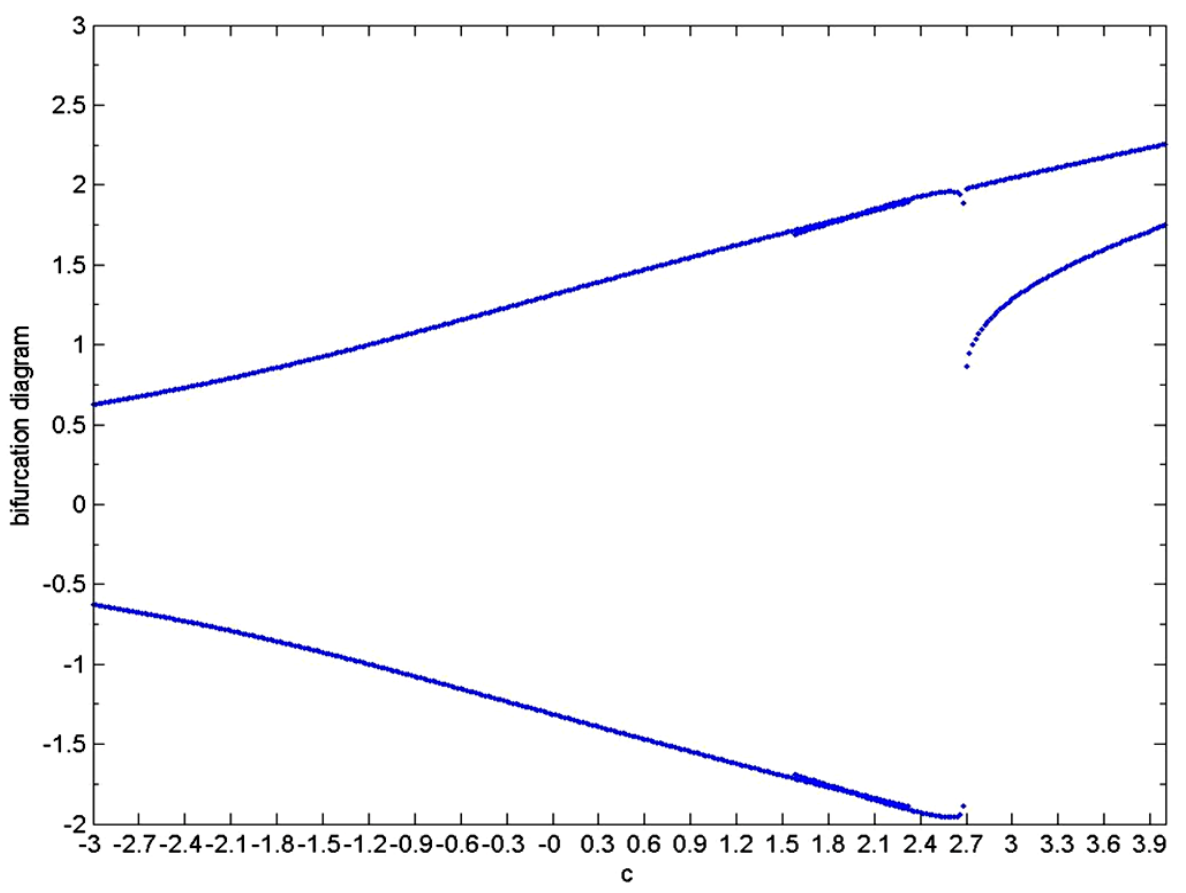

(a)

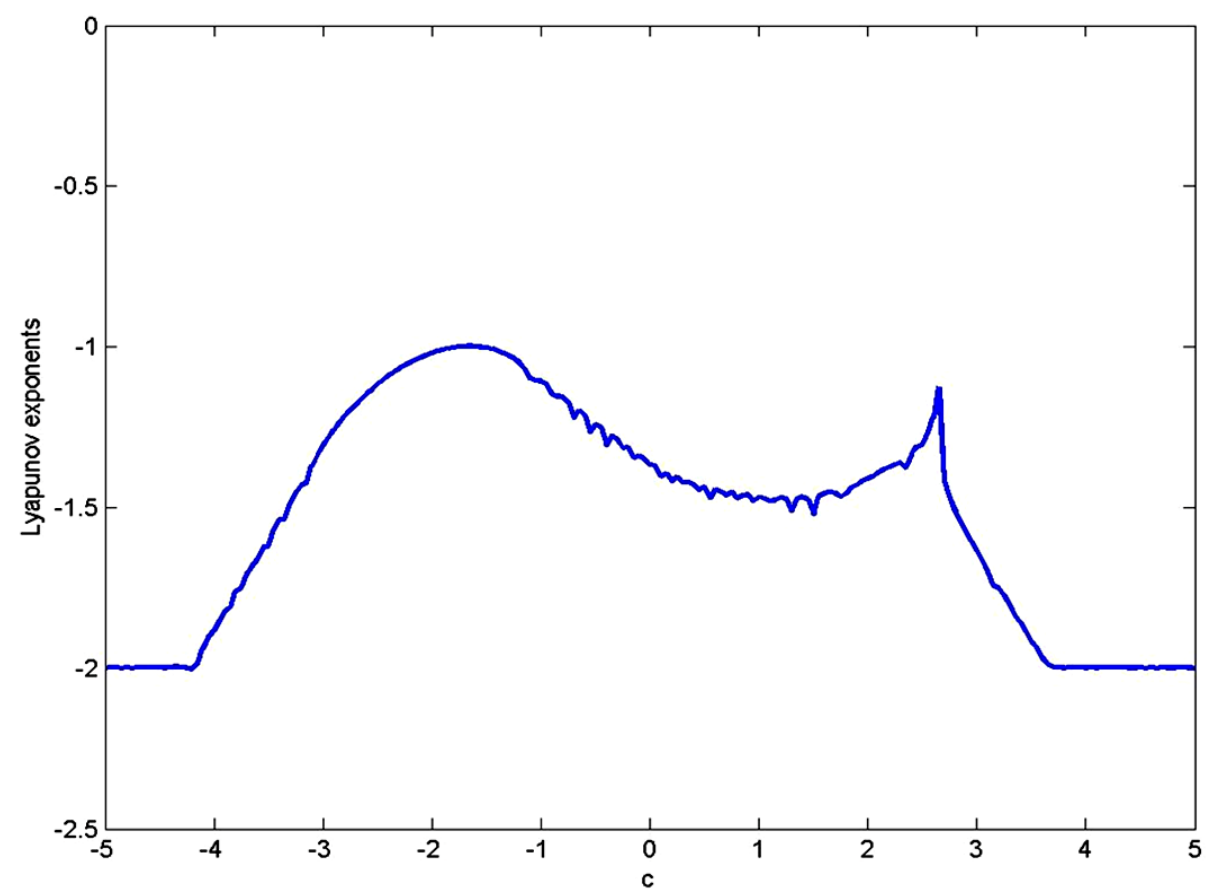

(b)

Figure 7. (a) Bifurcation diagram of $x \quad(c \in[0,4])$; (b) The maximum Lyapunov exponents corresponding to (a).

process of information transmission. We modified the system of Equation (1.2) into an actually practical one by adding the controller, which played the same role as damping. What's more, we discussed the sensitivity to be controlled and discovered the practical parameters regions. The control system will be more satisfactory in actual practice. 


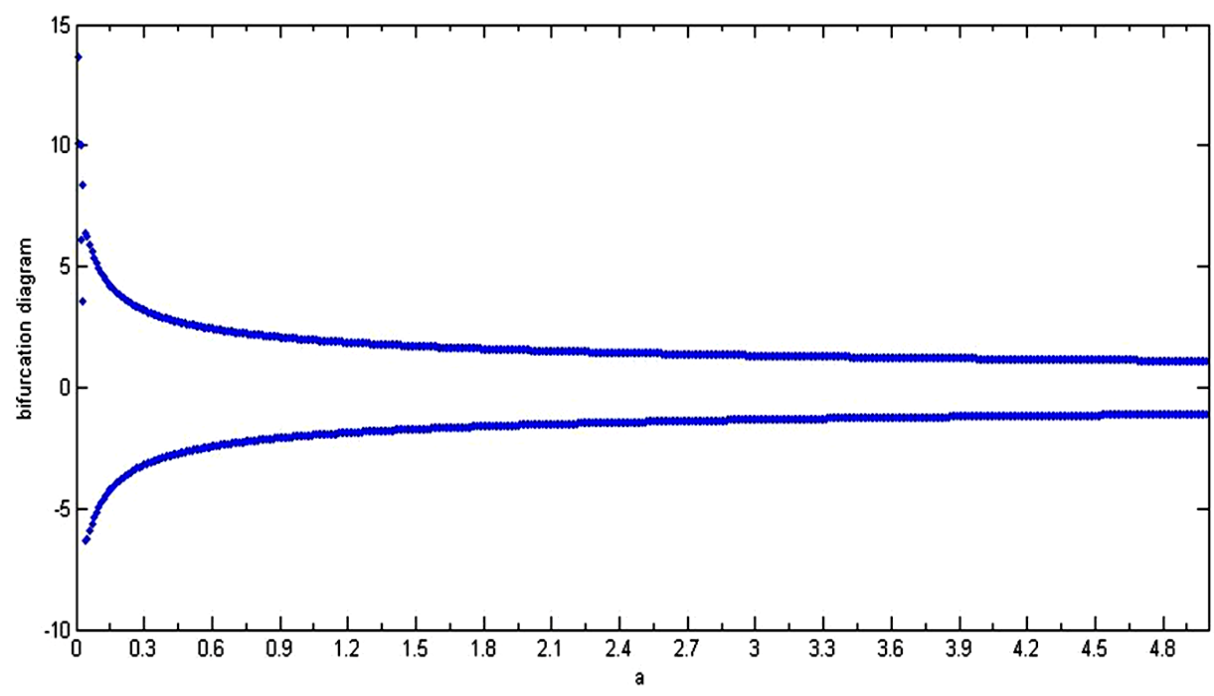

(a)

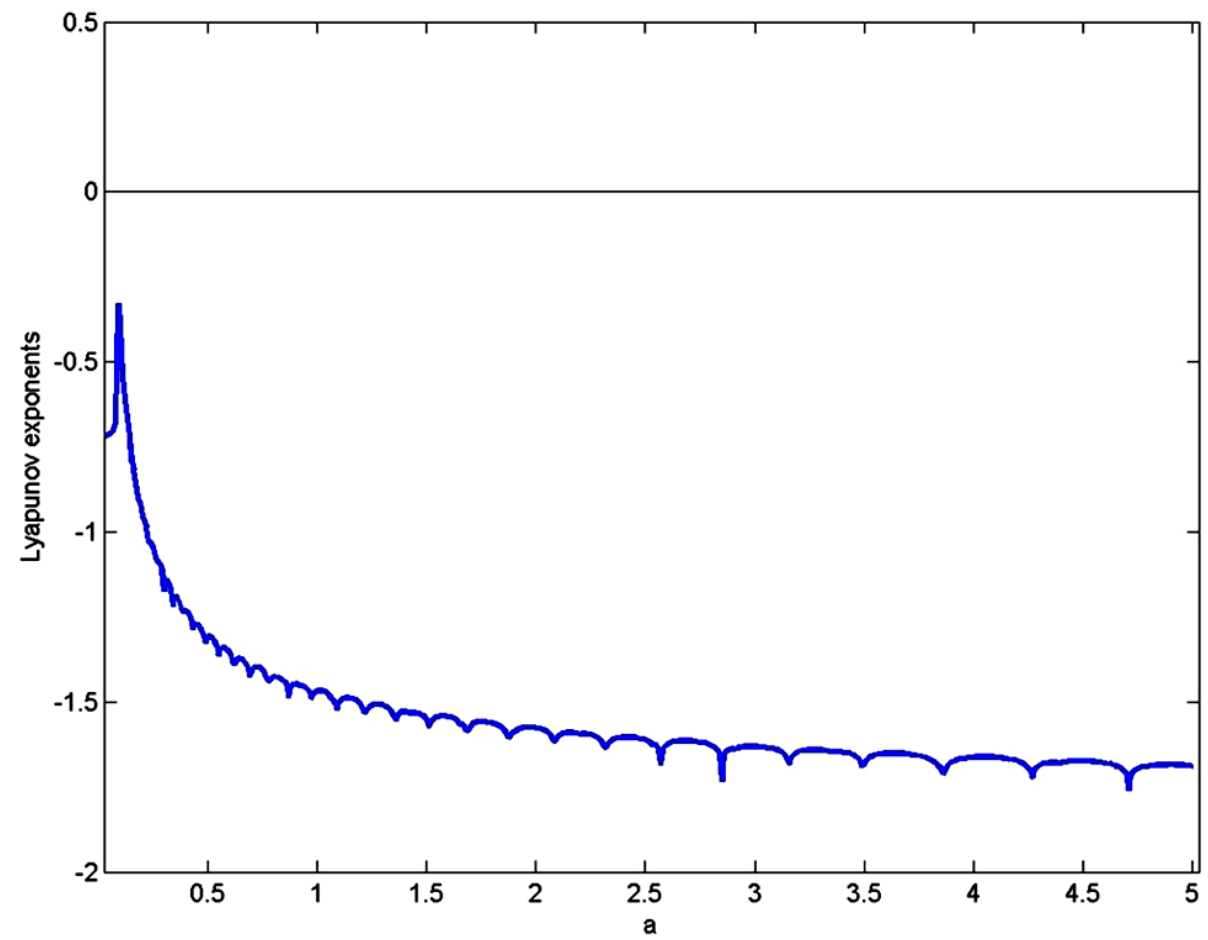

(b)

Figure 8. (a) Bifurcation diagram of $x \quad(a \in[0,5])$; (b) Maximum Lyapunov exponents corresponding to (a).

\section{Acknowledgements}

This work is supported by the National Nature Science Foundation of China (No. 11101191).

\section{References}

[1] de Bouard, A. and Debussche, A. (2010) The Nonlinear Schrödinger Equation with White Noise Dispersion. Journal of Functional Analysis, 259, 1300-1321. http://dx.doi.org/10.1016/j.jfa.2010.04.002

[2] Nakkeeran, K. and Wai, P.K.A. (2005) Generalized Projection Operator Method to Derive the Pulse Parameters Equa- 
tions for the Nonlinear Schrödinger Equation. Optics Communications, 244, 377-382. http://dx.doi.org/10.1016/j.optcom.2004.09.022

[3] Ndzana, F., Mohamadou, A. and Kofané, T.C. (2007) Modulational Instability in the Cubic-Quintic Nonlinear Schrödinger Equation through the Variational Approach. Optics Communications, 275, 421-428. http://dx.doi.org/10.1016/j.optcom.2007.02.036

[4] Hoseini, S.M. and Marchant, T.R. (2010) Evolution of Solitary Waves for a Perturbed Nonlinear Schrödinger Equation. Applied Mathematics and Computation, 216, 3642-3651. http://dx.doi.org/10.1016/j.amc.2010.05.015

[5] Dereli, Y., Irk, D. and Dag, I. (2009) Chaos, Soliton Solutions for NLS Equation Using Radial Basis Functions. Solitons and Fractals, 42, 1227-1233. http://dx.doi.org/10.1016/j.chaos.2009.03.030

[6] Korabel, N. and Aslavsky, G.M.Z. (2007) Transition to Chaos in Discrete Nonlinear Schrödinger Equation. Physica A, 378, 223-237. http://dx.doi.org/10.1016/j.physa.2006.10.041

[7] Shlizerman, E. and Rom-Kedar, V. (2006) Three Types of Chaos in the Forced Nonlinear Schrödinger Equation. Physical Review Letters, 96, Article ID: 024104. http://dx.doi.org/10.1103/PhysRevLett.96.024104

[8] Kivshar, Y.S. and Pelinovsky, D.E. (2000) Self-Focusing and Transverse Instabilities of Solitary Waves. Physics Reports, 331, 117-195. http://dx.doi.org/10.1016/S0370-1573(99)00106-4

[9] Korabel, N. and Zaslavsky, G.M. (2007) Transition to Chaos in Discrete Nonlinear Schrödinger Equation with LongRange Interaction. Physica A, 378, 223-237. http://dx.doi.org/10.1016/j.physa.2006.10.041

[10] Henning, D. and Tsironis, G.P. (1999) Wave Transmission in Nonlinear Lattices. Physics Reports, 307, $333-432$. http://dx.doi.org/10.1016/S0370-1573(98)00025-8

[11] Sharma, A., Patidar, V., Purohit, G., Sud, K.K. and Bishop, A.R. (2012) Effects on the Bifurcation and Chaos in Forced Duffing Oscillator Due to Nonlinear Damping. Communications in Nonlinear Science and Numerical Simulation, 17, 2254-2269. http://dx.doi.org/10.1016/j.cnsns.2011.10.032

[12] Jing, Z.J., Huang, J.C. and Deng, J. (2007) Complex Dynamics in Three-Well Duffing System with Two External Forcings. Chaos, Solitions \& Fractals, 33, 795-812. http://dx.doi.org/10.1016/j.chaos.2006.03.071

[13] Wang, R.Q., Deng, J. and Jing, Z.J. (2006) Chaos Control in Duffing System. Chaos, Solitons and Fractals, 27, 249257. http://dx.doi.org/10.1016/j.chaos.2005.03.038

[14] Stanton, S.C., Mann, B.P. and Owens, B.A.M. (2012) Melnikov Theoretic Methods for Characterizing the Dynamics the Bistable Piezoelectric Inertial Generator in Complex Spectral Environments. Physica D, 241, 711-720. http://dx.doi.org/10.1016/j.physd.2011.12.010

[15] Li, P., Yang, Y.R. and Zhang, M.L. (2011) Melnikov’s Method for Chaos of a Two-Dimensional Thin Panel in Subsonic Flow with External Excitation. Mechanics Research Communications, 38, 524-528. http://dx.doi.org/10.1016/j.mechrescom.2011.07.008 